\title{
Erectile dysfunction: Disease or symptom?
}

\section{Editorial}

Volume 2 Issue 3- 2021

\author{
Author Details \\ Simon Vallejo-Morales ${ }^{1}$ Yovany Cerinza-Garcia², Daniela Aldana-García ${ }^{3}$, Ivan David Lozada-Martinez \\ ${ }^{1}$ School of Medicine, Fundacion Universitaria San Martin, Colombia \\ ${ }^{2}$ School of Medicine, Universidad Nacional de Colombia, Colombia \\ ${ }^{3}$ School of Medicine, Universidad del Rosario, Colombia \\ ${ }^{4}$ School of Medicine, Universidad de Cartagena, Colombia
}

*Corresponding author

Ivan David Lozada-Martinez, School of Medicine, Universidad de Cartagena, Cartagena, Colombia

Article History

Received: September 14, 2021 Accepted: September 15, 2021 Published: September 16, 2021

\section{Editorial}

Erectile dysfunction remains a taboo today, despite the abundant evidence and dissemination of scientific knowledge accessible and understandable to the general community. From the social and cultural perspective it is mainly associated with lack of male virility, homosexuality, promiscuity or neuropsychiatric disorder, instead of giving it the correct argument, that of vascular aging and marker of accelerated cellular oxidation. For this reason, it is worth asking, is erectile dysfunction a symptom or a disease?

The European Association of Urology defines erectile dysfunction as the persistent inability to have and maintain an erection that allows satisfactory sexual intercourse [1]. Although there are other conditions that can lead to erectile failure, the vast majority of cases involve a pathophysiological process that may be intimately related to aging due to physiological oxidation or the negative impact of risk factors. Hypogonadism, decreased hormonal flow of testosterone and endothelial dysfunction are the pathophysiological basis of erectile dysfunction [1]. Vascular failure that does not allow adequate filling and maintenance of the volume of the corpora cavernosa is the first sign of endothelial dysfunction and physiological cardiovascular risk in men. So much so that studies have attempted to postulate this symptom as an early marker of other diseases associated with cardiovascular system involvement, such as chronic kidney disease [2].

Also, studies in murine models have evaluated the expression of specific genes in the corpora cavernosa for the early diagnosis of diabetes mellitus, showing that the genes Cyp1a1, Gclm, Igfbp5, Nqo1, Il6, Cxcl5, Olr1, Ctgf, Hbegf, Serpine1, Cyr61, Angptl4, and Loxl2 play a critical role in the signaling of insulin resistance and endothelial dysfunction [3]. Thus, the evidence supports that erectile dysfunction is indeed a symptom, but also a sign of cardiovascular risk.
However, the health education of the general population and the repercussions of popular beliefs influence the mental health of those affected [4-6]. Men who are victims of ridicule and rejection due to erectile dysfunction may develop neuropsychiatric disorders with severe complications such as self-harm and suicide [4-6]. Many of them turn to pseudosciences and alternative medicine in the hope of achieving satisfactory results. Therefore, although not directly a disease, erectile dysfunction may be a potential risk factor for developing neuropsychiatric diseases [4-6].

In this order of ideas, it is necessary to carry out educational plans and innovative strategies, especially in low and middle-income countries where traditional beliefs prevail that may threaten the integrity of evidence and men, with respect to this condition

\section{References}

1. Salonia A, Bettocchi C, Boeri L, Capogrosso P, Carvalho J, et al. (2021) European Association of Urology Guidelines on Sexual and Reproductive Health-2021 Update: Male Sexual Dysfunction. Eur Urol 80(3): 333-357.

2. Cirakoglu A, Yuce A, Benli E, Kasko Arici Y, et al. (2021) Is erectile dysfunction an early clinical symptom of chronic kidney disease? Aging Male 24(1): 24-28.

3. Yin GN, Ock J, Choi MJ, Limanjaya A, Ghatak K, et al. (2021) Gene expression profiling of mouse cavernous endothelial cells for diagnostic targets in diabetes-induced erectile dysfunction. Investig Clin Urol 62(1): 90-99.

4. Atallah S, Johnson-Agbakwu C, Rosenbaum T, Abdo C, Byers ES, et al. (2016) Ethical and Sociocultural Aspects of Sexual Function and Dysfunction in Both Sexes. J Sex Med 13(4): 591-606.

5. Irekpita E, Awe O, Salami T, Imomoh P, Oseni T (2017) Clinical, cultural and psychosocial impediments to self reporting of erectile 
dysfunction by men in Edo state, Nigeria. African Journal of Urology 23(2): 160-165.
6.

Bhavsar V, Bhugra D. Cultural factors and sexual dysfunction in clinical practice. Cambridge University Press. 